\title{
A SOA Web-based Group Decision Support System Considering Affective Aspects
}

\author{
Luís Conceição $^{1}$, João Carneiro ${ }^{1,2}$, Goreti Marreiros ${ }^{1}$ and Paulo Novais ${ }^{2}$ \\ ${ }^{1}$ GECAD - Research Group on Intelligent Engineering and Computing for Advanced Innova- \\ tion and Development, Institute of Engineering, Polytechnic of Porto, Porto, Portugal \\ \{lmdsc, jomrc, mgt\} @isep. ipp.pt \\ ${ }^{2}$ ALGORITMI Centre, University of Minho, Braga, Portugal \\ pjon@di.uminho.pt
}

\begin{abstract}
The topic of Group Decision Support Systems (GDSS) has been studied over the last decades. Supporting decision-makers that participate in group decision-making processes is a complex task, especially when decision-makers have no opportunity to gather at the same place and at the same time. In this work, we propose a Web based Group Decision Support System (WebGDSS) which intends to support decision-makers anywhere, anytime and through almost any kind of devices. Our system was developed under a SOA architecture and we used a multi criteria algorithm that features decision-makers' cognitive aspects, as well as a component of generation of intelligent reports to feedback the results of decision-making processes to the decision-makers.
\end{abstract}

Keywords: Group Decision Support Systems, Group Decision-Making, Intelligent Reports, Cognitive Decision-Making.

\section{Introduction}

Nowadays, group decision-making is the most preferred way of making decisions in companies around the world. It's known that when decisions are made in group, better results are achieved [1].

Group Decision Support Systems (GDSS) have been studied throughout the last decades with the objective of supporting decision-makers in group decision-making processes. The number of participants in a group decision-making process is variable and each participant may be at the same place at the same time or may be in different countries and with different time zones, rending a group decision-making process is a challenge quite difficult to tackle [2].

GDSSs have seen quite an evolution since their introduction [3]. This is mainly attributable to the evolution of general technology, as well as to the need of enhancing the efficiency of the group decision-making processes.

There is quite a large number of GDSSs relevant presented in literature [4-9]. However, the success of these systems hasn't been positive because the organizations' acceptance of these systems has been low due to numerous factors. On the one hand, several of the existing systems feature interfaces too complex that won't be filled in by 
the busy decision-maker, which is what enables the decision-maker to reflect the respective preferences regarding the available alternatives. On the other hand, some of the systems do not allow decision-makers to properly express their preferences [10] or do not fully transmit the decision-maker's opinion to the system, resulting in a loss of relevant information for the decision-making process.

In this paper, we propose a Web Based Group Decision Support System capable of aiding groups of decision-makers in decision-making processes, namely in multi criteria problems. The proposed system has been developed using a Services-oriented Architecture (SOA) to help mainstream the integration of the application in several platforms (eg: PCs, tablets, smartphones) which, not only does it feature decision-makers' cognitive aspects, it has a problem configuration interface that simplifies the whole process and allows decision-makers to express their preferences, based on the work of $[10,11]$. Moreover, the present GDSS features a component of generation of intelligent reports so that the relevant decision-maker finds the information provided throughout the several iterations of the decision-making process useful, aiming to meet the decision-maker's interests in that same process, while trying to maximize his/her approval of the final decision.

The rest of the paper is organized as follows: in Section 2 we describe some existing works in literature. Section 3 describes our proposed GDSS, namely its functionalities and architecture, and in Section 3 we present some conclusions and some guidelines regarding future work that we aim to carry on.

\section{State of the Art}

We did an analysis of some existing GDSSs in the literature in order to analyze the software architectures, their functionalities and applications.

In [7], authors presented a scientific paper where they developed a scenario of simulated medical practice for intelligent support for decision making on the stages of cancer. Decisions were made in the context of a group meeting to facilitate collaborative work. The authors used agents to represent real participants and to exchange and store information. The developed system emulated the phases of cancer, allowing to increase the performance of the medical team and eliminate the circulation of paper.

In [9] authors have developed a web based GDSS that allows support groups of decision makers in group decision making processes. The developed GDSS allows a facilitator to create a decision problem, its alternatives and add the experts who will participate in the decision process. In this system experts only introduce their preferences to alternatives. These preferences are indicated in the form of comparison of the alternatives with each other. Later, at each iteration of the decision process, the computation module evaluates the preferences introduced by the experts and makes suggestions if there are inconsistencies between the preferences.

Another interesting work is the one's presented in [6]. In this work authors have developed a model of a GDSS that uses agents to represent the participants of a meeting. In this approach, the agents were not meant to replace the members of the meeting, but rather the intention to support them in the group decision-making process. In this work, 
the agents are endowed with an emotional component due to the importance that the emotions have in the negotiation process. The architecture of the participating agents is composed of three layers: Knowledge, Reasoning and Interaction.

In [8], authors bring to us a very relevant and practical work where they proposed a GDSS for the evaluation of alternative pipelines routes to transport oil and natural gas from Caspian Sea to others distant regions. They decompose the route selection process into manageable steps. They combine Strength, Weakness, Opportunity and Threat (SWOT) analysis with the Delphi method to capture the decision-makers' beliefs. They also developed a model called Preference Ranking Organization Method for Enrichment Evaluation (PROMETHEE) to integrate the decision-makers' beliefs with subjective judgments and identify the most attractive pipeline route. They claim that their system encourages decision-makers to think systematically and carefully consider environmental complexities and uncertainties. They see as future challenges the incorporation of simulating and optimization methods in the GDSS.

A more recent work published by [5] where they presented an experimental study of a new web-based called Group Remote Asynchronous Screening Support (GRASS). They used interactive decision maps/reasonable goals method in remote asynchronous group decision-making. They used reasonable goals method where an individual can select a small number of alternatives for subsequent ranking. They claim that the GRASS produces better results when used by someone familiar with the procedure. However, the non-experienced people failed to use GRASS without the help of an instructor.

In [12] addressed a very interested work about how GDSS can facilitate and help the organizations. They performed a good literature review about this thematic and did an experiment to test whether the use of a GDSS increases the quality of a brainstorm sessions. Besides they consider that GDSS promises very potential, they did not find any evidences to say that GDSS increased the quality of brainstorm results.

\section{Proposed GDSS}

The system recommended in this paper has the objective of being the most ubiquitous system so that it supports decision-makers anytime, anywhere and from any device or equipment, as long as it has an Internet connection.

The developed GDSS aims to aid groups of decision-makers in multi criteria decision-making processes. In this system, each participant configures his/her preferences regarding the problem and, after every participant has introduced the respective preferences, the system runs the decision algorithm and presents the results in intelligent reports. 


\subsection{System Architecture}

The system architecture (Fig. 1) was designed in such a way that it allows for the usage of the system in any device (e.g. PCs, tablets, smartphones) due to the implementation of the Service-oriented Architecture (SOA) methodology. This way, it is possible to easily develop applications for different devices with different technologies, enabling decision-makers to use the GDSS in any type of device, anytime and from anywhere, as long as they have an Internet connection.

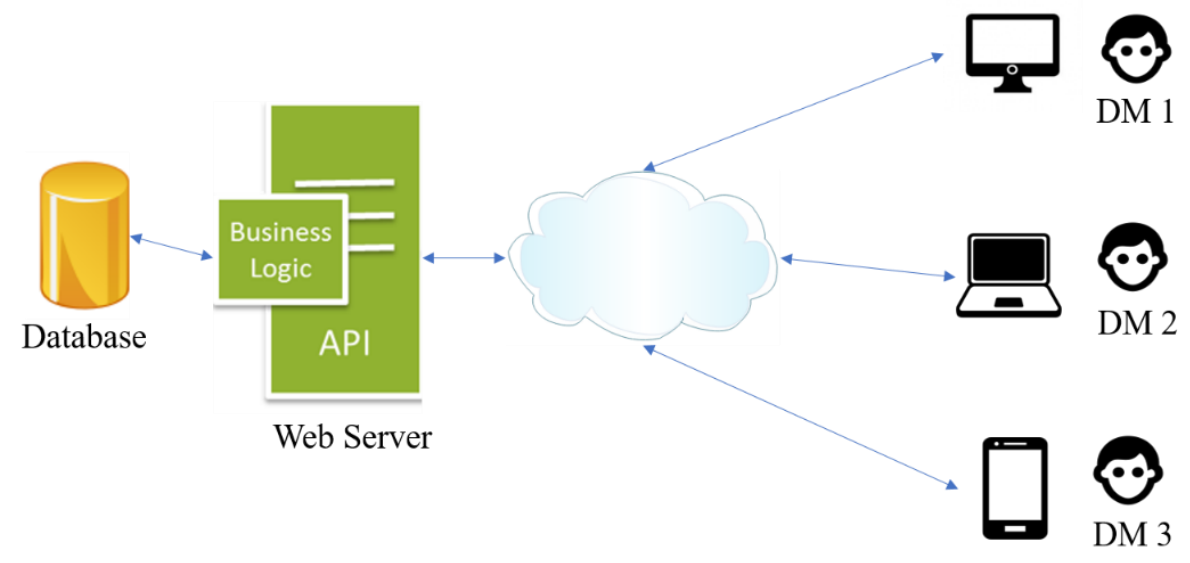

Fig. 1. System Architecture

The system offers a Application Programming Interface (API) that implements business logic, responsible for modulating the actions allowed by the application, so that the client applications only need to implement interfaces with the end user (the decision-maker).

The API implements all the system's business layers, as well as the control of the access to the database, implementing all functions of Create, Read, Update and Delete (CRUD), where all GDSS information is stored. Besides this, it implements the multi criteria decision-making algorithm. In this specific case, the chosen algorithm is the Cognitive Analytic Process (CAP) [13].

CAP is an algorithm for the sorting of alternatives within multi criteria problems that features decision-makers' cognitive aspects. This algorithm evaluates alternatives and criteria preferences indicated by each decision-maker, but it goes beyond that: it takes into account the intentions of the decision-makers. The CAP considers the behaviour style and the expertise level of the decision-maker and it also makes use of the information of which decision-makers are considered knowledgeable. All this information is processed by the CAP, presenting a solution that combines the rational component with the irrational one [13].

The developed API implements authentication services in order to ensure data integrity and security, as well as every service of manipulation of the decision-making problems, alternatives, criteria, etc. It is possible to access all system functionalities through 
the API (e.g. creating problems, criteria, alternatives, and selecting which decisionmakers will participate in a given problem, etc).

\subsection{Web Application}

The Web Apllication implements user interfaces between the GDSS and the decisionmaker. This application allows the user to configure the multi-criteria problem, namely their alternatives and the criteria that evaluate each of the alternatives. In addition to the alternatives and criteria, the user who creates the problem must also add the users who will participate in that decision-making process. The preferences configuration interface was defined according to the work proposed by $[10,11]$, Where it presents a multi-criteria system configuration template for a multi-agent system. The results of the system are presented through a intelligent report generation feature $[14,15]$ which reports to each decision-maker only the information considered relevant according to their profile in the decision-making process.

\subsection{System Functionalities}

The primary objective of a GDSS is to support a group of decision-makers in a group decision-making process.

The user intending to start a decision-making process (the organizer) has to set up the problem and for that he has to:

1. Define the problem for discussion;

2. Define the criteria to consider;

3. Define the available alternatives;

4. Select the participating decision-makers.

The organizer needs to have all this information previously defined so that he/she is able to input them into the system. This information is usually gathered during brainstorming meetings, or by other methods, depending on the problem for which a decision is being made. In GDSS, the creation of a new problem prompts the user for a problem title and brief description.

The next step is the creation of criteria, which can be Boolean, Numeric or Classificatory. In case they are of the Classificatory type, the organizer has to define a numerical value for each of the classifications. Besides the type of data that measure the criterion, it is necessary to indicate if the criterion is of minimization or maximization type (e.g. if a given criterion is the buying price of a given product, and considering that the intention is to minimize the cost of that purchase, the criterion Price will be a minimization criterion).

Once the problem criteria are set up, the next task is to define the alternatives that may be the solutions for the problem. Each alternative has a name, a field for a brief 
description, and its evaluation based on the criteria defined in the previous stage. Therefore, for each alternative, the organizer has to specify the value of that alternative in each of the defined criteria.

After inserting the alternatives and the criteria, the organizer is left with the task of adding the decision-makers that will participate in the decision-making process, as well as setting a deadline for the process and finalizing the problem creation.

Upon completion of these actions, decision-makers selected to participate in the process will be alerted via email that a new problem has been created and that their intervention is needed to indicate their preferences regarding the alternatives and criteria to be considered.

The decision makers who will participate in the process have access to the interface of configuring their preferences through the link received via email. Each decision maker has now the possibility to configure the preferences of the system on the problem to be discussed. First, begin by setting personal preferences for the problem as shown in the Fig. 2. In this case, each decision-maker can indicate his / her Expertise Level in relation to the issue, the behavior style that he/she wants, and which decision-makers he or she considers to be credible (within the group of participants).

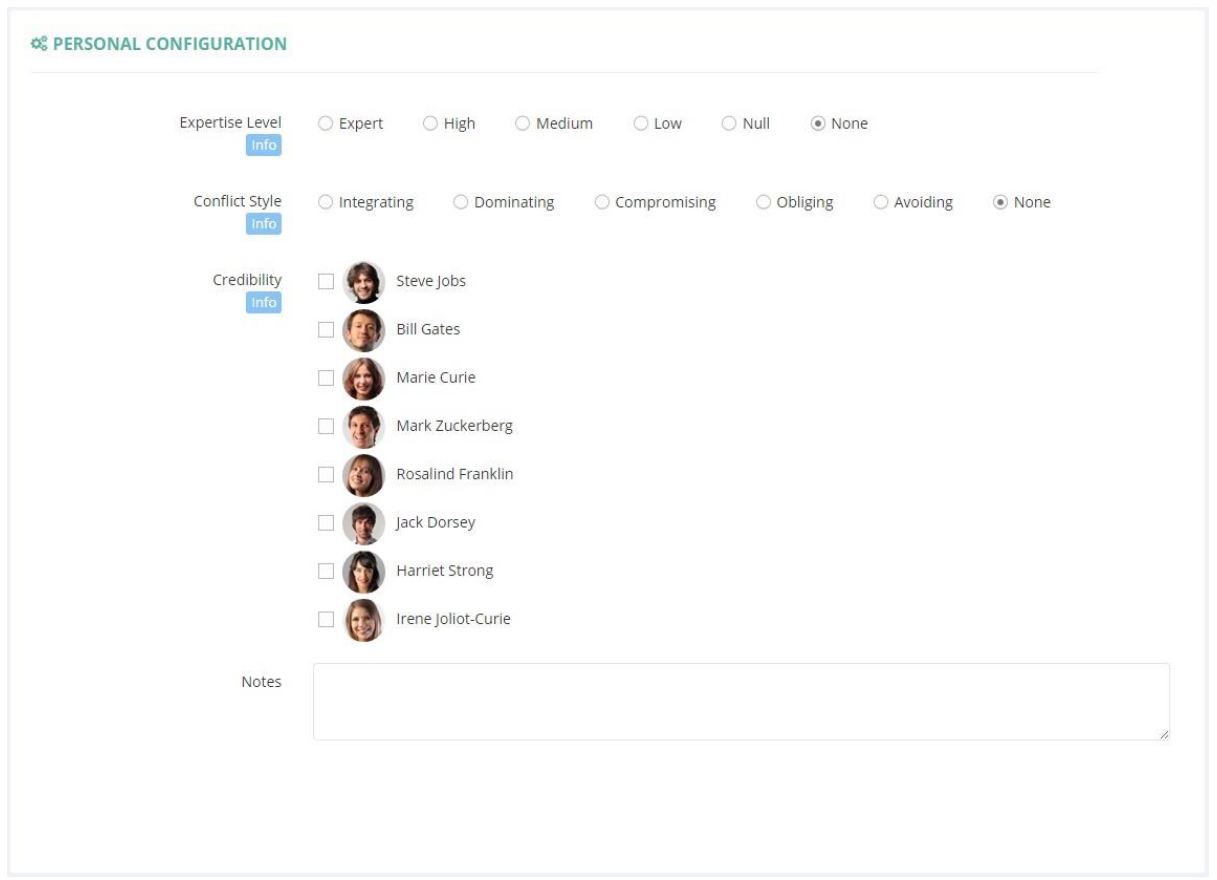

Fig. 2. Personal Configuration

The Expertise Level intends to represent the level of expertise of the decision maker in relation to the problem being discussed (to read more about this see [13]). 
Credibility is related to the decision maker's perception of the credibility of the other participants in the process in relation to their expertise regarding the problem being discussed (to read more about this see [13]).

Behavior style can be seen as the desired behavior or behavior of the system in the "defense" of the interests/preferences of the decision maker during the decision-making process (to read more about this see [16]). Available styles (presented in Table 1) are: Dominating, Integrating, Compromising, Obliging and Avoiding. These styles differentiate each other through 4 dimensions:

- Concern for Self - This dimension is related to the individual's concern for his own opinion above the others since he is likely to adapt a more one-sided attitude during the decision-making process by making statements, questions and requests that detail that opinion;

- Concern for others - This dimension relates to the individual's concern for other individuals' opinion. He adapts a more altruist attitude during the decision-making process, trying to understand other opinions and making an effort to reach a decision that benefits or pleases most of the participants;

- Activity - This dimension relates to the effort put into the decision-making process by the individual, meaning that the more active an individual is, the more questions and statements and requests he is likely to make;

- Resistance to change - This dimension relates to how hard or easy it is for an individual to accept other opinions.

Table 1. Behaviour Styles and corresponding dimensions, adapted from [16]

\begin{tabular}{lllll}
\hline Conflict Style & $\begin{array}{l}\text { Concern For } \\
\text { Self }\end{array}$ & $\begin{array}{l}\text { Concern for } \\
\text { Others }\end{array}$ & Activity & $\begin{array}{l}\text { Resistance to } \\
\text { Change }\end{array}$ \\
\hline Dominating & High & Low & High & High \\
Integrating & High & High & High & High \\
Compromising & Moderate & Moderate & Moderate & Moderate \\
Obliging & Low & High & Low & Low \\
Avoiding & Low & Low & Low & Low \\
\hline
\end{tabular}

After configuring personal information, each decision-maker has to indicate their preferences regarding the attributes of the problem: the criteria and the alternatives. Preferences are indicated on a scale of 0 to 100 as shown in Fig. 3.

When all the decision-makers involved in the process configure their preferences, the system executes the algorithm and concludes the process. This process is iterative until all members of the group agree on the final decision, or until the organizer completes the process. In the event that a decision maker does not accept the decision, it can readjust its preferences and then the process returns to the beginning.

The information is reported in the form of intelligent reports as presented in Fig. 4. Intelligent reports have the objective of reporting to each decision-maker only the information that is considered relevant according to their position (their interests) in each 
group decision-making process. With this functionality, we intend to report to the decision-makers brief and relevant information so that each decision-maker can analyze the results of each iteration of the process by spending as little time as possible (to read more about this see $[14,15])$.

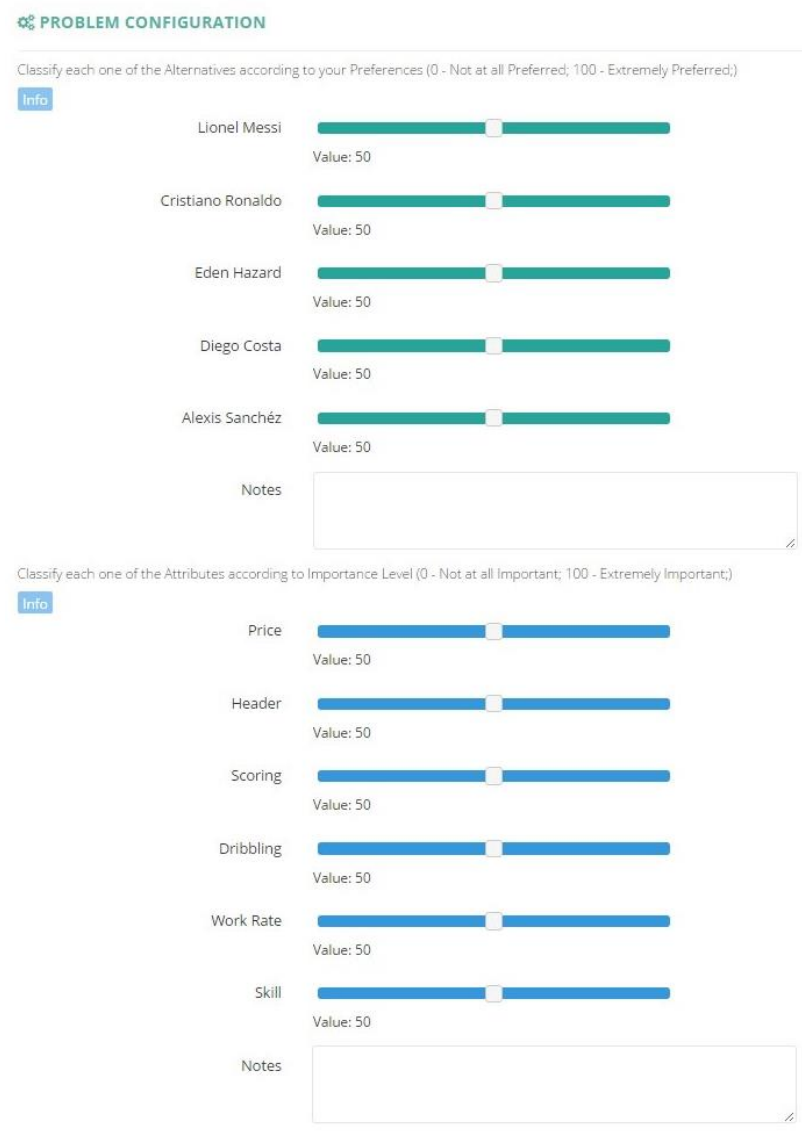

Fig. 3. Problem Configuration 


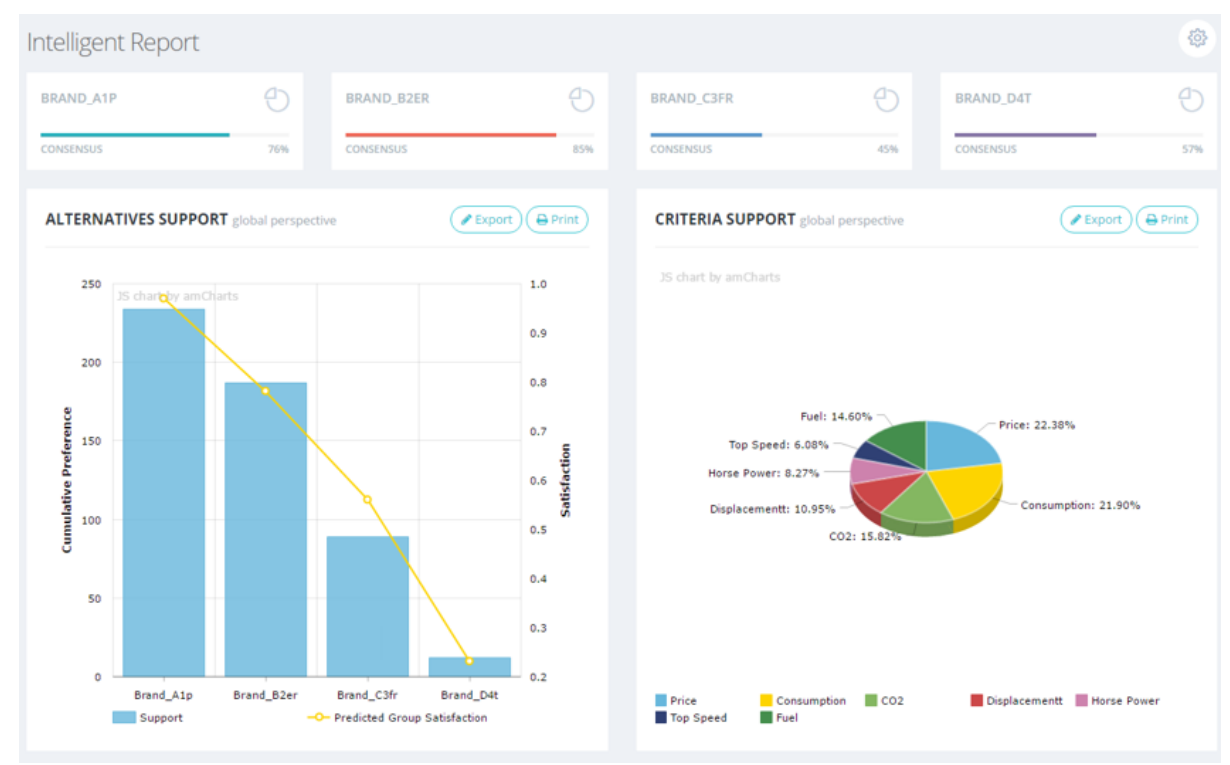

Fig. 4. Intelligent Report

\section{Conclusions and Future Work}

GDSS have become an important topic of study in recent decades. Its benefits and its advantages are recognized by all, especially when it comes to supporting decision makers who are not in the same place. Despite this, we know that these systems have not been well accepted by organizations. As we mentioned in our previous works [10, 14], the interaction between decision-makers and the system is an essential point regarding the acceptance of systems by users and the organizations themselves.

The development of this system is the result of extensive research related to group decision making. Our proposal aims to fill some of the flaws identified in the literature, especially in relation to usability factors. In this work, we developed a web-based GDSS where the results of previous works are applied, with the aim of presenting a system that allows the groups of decision makers to make decisions remotely without the relevant aspects such as the personality and the influence of the relations between the elements of the group being lost.

As future work, we intend to develop a case study with a real group of decisionmakers in a real-life context in order to validate the system.

\footnotetext{
Acknowledgments. This work was supported by GECAD - Research Group on Intelligent Engineering and Computing for Advanced Innovation and Development and by National Funds through the FCT - Fundação para a Ciência e a Tecnologia (Portuguese Foundation for Science and Technology) with the João Carneiro Ph.D. Grant with the Reference SFRH/BD/89697/2012.
} 


\section{References}

1. Luthans, F.: Organizational behavior. McGraw-Hill/Irwin 46, 594 (2011)

2. Huber, G.P.: Issues in the Design of Group Decision Support Systems. MIS Quarterly: Management Information Systems 8, 195-204 (1984)

3. DeSanctis, G., Gallupe, B.: Group decision support systems: a new frontier. SIGMIS Database 16, 3-10 (1985)

4. Choi, D.-Y.: Aggregation of Preferences Based on FSAM in GDSS. Systems, Man and Cybernetics, Part A: Systems and Humans, IEEE Transactions on 38, 2-8 (2008)

5. Efremov, R.V., Lotov, A.V.: Multi-criteria remote asynchronous group decision screening: an experimental study. Group Decision and Negotiation 23, 31-48 (2014)

6. Marreiros, G., Santos, R., Ramos, C., Neves, J.: Context-Aware Emotion-Based Model for Group Decision Making. Intelligent Systems, IEEE 25, 31-39 (2010)

7. Miranda, M., Abelha, A., Santos, M., Machado, J., Neves, J.: A group decision support system for staging of cancer. Electronic Healthcare, pp. 114-121. Springer (2008)

8. Tavana, M., Behzadian, M., Pirdashti, M., Pirdashti, H.: A PROMETHEE-GDSS for oil and gas pipeline planning in the Caspian Sea basin. Energy Economics 36, 716-728 (2013)

9. Alonso, S., Herrera-Viedma, E., Chiclana, F., Herrera, F.: A web based consensus support system for group decision making problems and incomplete preferences. Information Sciences 180, 4477-4495 (2010)

10.Carneiro, J., Santos, R., Marreiros, G., Novais, P.: Overcoming the Lack of HumanInteraction in Ubiquitous Group Decision Support Systems. In: Advanced Science and Technology Letters, pp. 116-124. (Year)

11.Carneiro, J., Martinho, D., Marreiros, G., Novais, P.: A General Template to Configure MultiCriteria Problems in Ubiquitous GDSS. International Journal of Software Engineering and Its Applications 9, 193-206 (2015)

12.Monteban, J.: Using Group Decision Support Systems to Facilitate Organizational Change. (2014)

13.Carneiro, J., Conceição, L., Martinho, D., Marreiros, G., Novais, P.: Including cognitive aspects in multiple criteria decision analysis. Annals of Operations Research 1-23 (2016)

14.Carneiro, J., Conceição, L., Martinho, D., Marreiros, G., Novais, P.: Intelligent reports for group decision support systems. In: Intelligent Environments 2016: Workshop Proceedings of the 12th International Conference on Intelligent Environments, pp. 4. IOS Press, (Year)

15.Conceição, L., Carneiro, J., Martinho, D., Marreiros, G., Novais, P.: Generation of intelligent reports for ubiquitous group decision support systems. In: Global Information Infrastructure and Networking Symposium (GIIS), 2016, pp. 1-6. IEEE, (Year)

16.Martinho, D., Carneiro, J., Marreiros, G., Novais, P.: Dealing with Agents' Behaviour in the Decision-Making Process. Workshop Proceedings of the 11th International Conference on Intelligent Environments 19, 4 (2015) 